International Journal of Pure and Applied Physics.

ISSN 0973-1776 Volume 14, Number 2 (2018), pp. 125-129

(C) Research India Publications

https://dx.doi.org/10.37622/IJPAP/14.2.2018.125-129

\title{
Effect of Donor/Acceptor group position on the NLO properties of branched chain organic molecules
}

\author{
Vijaya Kumari ${ }^{\mathbf{a}, b^{*}}$ \\ ${ }^{a}$ Shri Madhwa Vadiraja Institute of Technology and Management, Vishwothama \\ Nagar, Bantakal, Udupi -574115, Karnataka, India. \\ ${ }^{b}$ Shri Bhuvanendra College, Karkala -574104, Karnataka, India.
}

\begin{abstract}
The organic materials are the most investigated materials for their nonlinear optical properties in the past three decades. In this article the systematic investigation on the NLO properties of Donor/Acceptor substituted branched chain organic chromophores, chalcones, using semi empirical method is presented. The nonlinear optical molecular static first hyperpolarizability $(\beta)$ was found to be larger for Donor/Acceptor group substituted at para position than that of substitution of Donor/Acceptor group at meta and ortho position. For designing NLO materials with high $\beta$ it is recommended to make substitution of Donor/Acceptor group at para position rather than meta and ortho position.
\end{abstract}

Keywords: nonlinear optics, Organic materials, molecular hyperpolarizability

\section{INTRODUCTION}

Nonlinear optical (NLO) materials are attractive due to their applications in optical frequency converter, optical switch, optical parametric oscillators etc [1,2]. Initially inorganic materials were explored for the NLO properties and they are adopted in NLO applications [3]. Due to lesser magnitude of NLO coefficients and slow response of inorganics, researchers started to explored new materials for NLO applications. In the search for new materials for NLO applications organic materials were found to be attractive due to ultrafast response time, structural flexibility, thermal and chemical stability. Among the different types of organic materials chalcones are notable materials due to their transparency in blue region, good crystallizability, high second harmonic generation(SHG) efficiency, third order NLO properties, high laser damage threshold and thermal stability [4,5]. The chalcones molecules can be designed possess either D- $\pi$-A type structure or D- $\pi$-A- $\pi$-D type structure. The donor group can be placed at the either ends of the molecule to obtain D- $\pi$-A- $\pi$-D structure with carbonyl group acting as an acceptor group at the center of the molecule. In order to design D- $\pi$-A type push-pull system in chalcones it is 
sufficient to substitute one donor group at the one end of the molecule or an acceptor group at the end.

In this article the investigation on the NLO properties of six chalcones molecules possessing D- $\pi$-A type push-pull structure using semi empirical quantum chemical calculations using MOPAC2016 software at AM1 level of theory. The molecular structures of all the six molecules were optimized and NLO properties were computed by semi empirical method. The effect of position of Donor/Acceptor group substitution on NLO properties of these branched chain chalcones is presented.

\section{COMPUTATIONAL PROCEDURE}

The NLO properties of Donor/acceptor substituted chalcones were computed using semi-empirical MOPAC 2016 package [6]. To optimize the molecular geometry of all the six molecules AM1/EF level of theory was adopted. PRECISE key word was used in geometry optimization in order improve the accuracy. To compute static molecular First hyperpolarizability $\beta_{\mathrm{o}}$ Time Dependent Hartree-Fock (TDHF) method was implemented. All the graphics were generated using Winmostar software.

\section{RESULT AND DISCUSSION}

The D- $\pi$-A type six push-pull structures of Chalcones namely $(1 E, 4 E)-1-(4-$ nitrophenyl)-5-phenylpenta-1,4-dien-3-one (PN), (1E,4E)-1-(3-nitrophenyl)-5phenylpenta-1,4-dien-3-one (MN), (1E,4E)-1-(2-nitrophenyl)-5-phenylpenta-1,4-dien3-one $(\mathrm{ON}), \quad(1 E, 4 E)-1$-(4-methoxyphenyl)-5-phenylpenta-1,4-dien-3-one (PM), (1E,4E)-1-(3-methoxyphenyl)-5-phenylpenta-1,4-dien-3-one (MM) and (1E,4E)-1(2-methoxyphenyl)-5-phenylpenta-1,4-dien-3-one (OM), were designed and the molecular structures are shown in Figure 1. In compound PM, MM and OM, Methoxy group attached to phenyl group acts as an electron donor whereas the carbonyl group along with the phenyl group at the other end acts as an electron withdrawing group. In compound PN, MN and ON the nitro phenyl along with carbonyl group acts as an electron withdrawing group and the phenyl group at the other end acts as an electron donor.
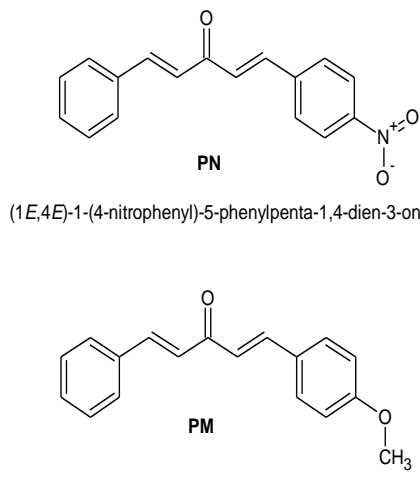

(1E,4E)-1-(4-methoxyphenyl)-5-phenylpenta-1,4-dien-3-one
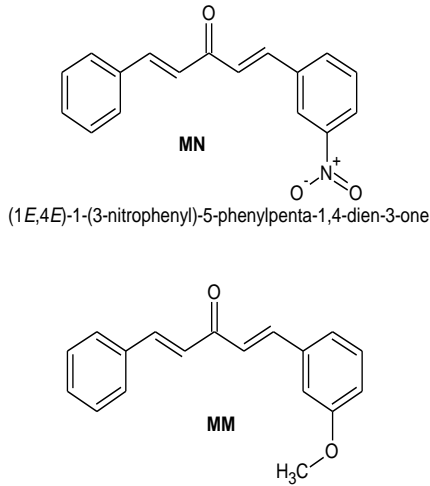

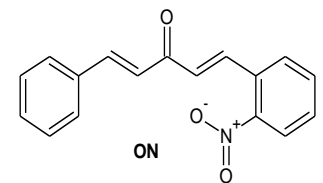

(1E,4E)-1-(2-nitrophenyl)-5-phenylpenta-1,4-dien-3-one

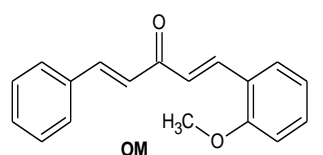

(1E,4E)-1-(2-methoxyphenyl)-5-phenylpenta-1,4-dien-3-one

Figure 1. Molecular structures of Donor/Acceptor substituted chalcones 
Table 1. Semi empirical quantum chemical computational results of Donor/Acceptor substituted chalcones

\begin{tabular}{|c|c|c|c|c|c|c|c|}
\hline Compound & $\begin{array}{c}\boldsymbol{\mu} \\
\text { (Debye) }\end{array}$ & $\begin{array}{l}\beta_{0}(\mathrm{X} 10 \\
\left.{ }^{30}\right) \text { esu }\end{array}$ & HOMO $(\mathrm{eV}$ & LUMO $(\mathrm{eV})$ & $\operatorname{Eg}(e V)$ & $\begin{array}{l}\text { DIHEDRAL } \\
\text { ANGLE }\end{array}$ & $\begin{array}{c}\mathbf{C}=\mathbf{C} \text { of } \\
\text { substitution } \\
\text { side }(\AA)\end{array}$ \\
\hline PM & 3.815 & 10.03 & -8.827 & -0.786 & 8.041 & 14.37 & 1.344 \\
\hline MM & 2.970 & 5.31 & -9.039 & -0.853 & 8.186 & 18.68 & 1.343 \\
\hline OM & 3.767 & 4.82 & -8.911 & -0.688 & 8.223 & 19.08 & 1.3428 \\
\hline PN & 5.681 & 8.32 & -9.469 & -1.582 & 7.887 & 34.87 & 1.3422 \\
\hline MN & 3.014 & 6.45 & -9.380 & -1.336 & 8.044 & 15.02 & 1.3423 \\
\hline ON & 6.582 & 6.67 & -9.340 & -1.315 & 8.025 & 76.73 & 1.3414 \\
\hline
\end{tabular}

The computational results are given in Table 1. It is clear from the data presented in Table 1 that the para position is the best position for Donor/Acceptor substitution to enhance the static first hyperpolarizability $\left(\beta_{0}\right)$. The $\beta_{0}$ values varies as $\mathrm{PM}>\mathrm{MM}>$ $\mathrm{OM}$ in the case of Methoxy substituted Chalcone, whereas $\mathrm{PN}>\mathrm{ON}>\mathrm{MN}$ in Nitro group substituted chalcone. Similar trend was observed in Energy gap of the computed molecules. Further the dihedral angle is more in OM in comparison with $\mathrm{MM}$ and PM. This suggest that the planarity of the molecule is better in MM . In addition the $\mathrm{C}=\mathrm{C}$ bond length is found to be more for $\mathrm{PM}$ which reflects the effectiveness of charge transfer in PM than that of OM and MM. The energy gap in Acceptor substituted compounds is found to be small for PN than that of MN and ON. Usually materials with smaller energy gap show better NLO property. The value of $\beta_{0}$ was found to be larger for the molecules in which the Donor/Acceptors substituted at para position.

In order to understand the nature of charge transfer the frontiers of molecular orbitals were computed and graphics were created with Winmostar. All the molecules in which electron with drawing group $(\mathrm{C}=\mathrm{O})$ and Donor/acceptor (Methoxy/Nitro) is connected via $\mathrm{C}=\mathrm{C}$. The frontier of molecular orbitals shown in Figure 3 clearly indicates that the charge transfer occur between methoxy phenyl group to carbonyl group. Whereas in Nitro substituted molecules phenyl group acts as a donor and Nitro group act as electron acceptor which are clearly reflected in Figure 2. The computational result, unambiguously reveal that to design chalcones for NLO application one need to preferably make substitution of donor/acceptor at para position. 


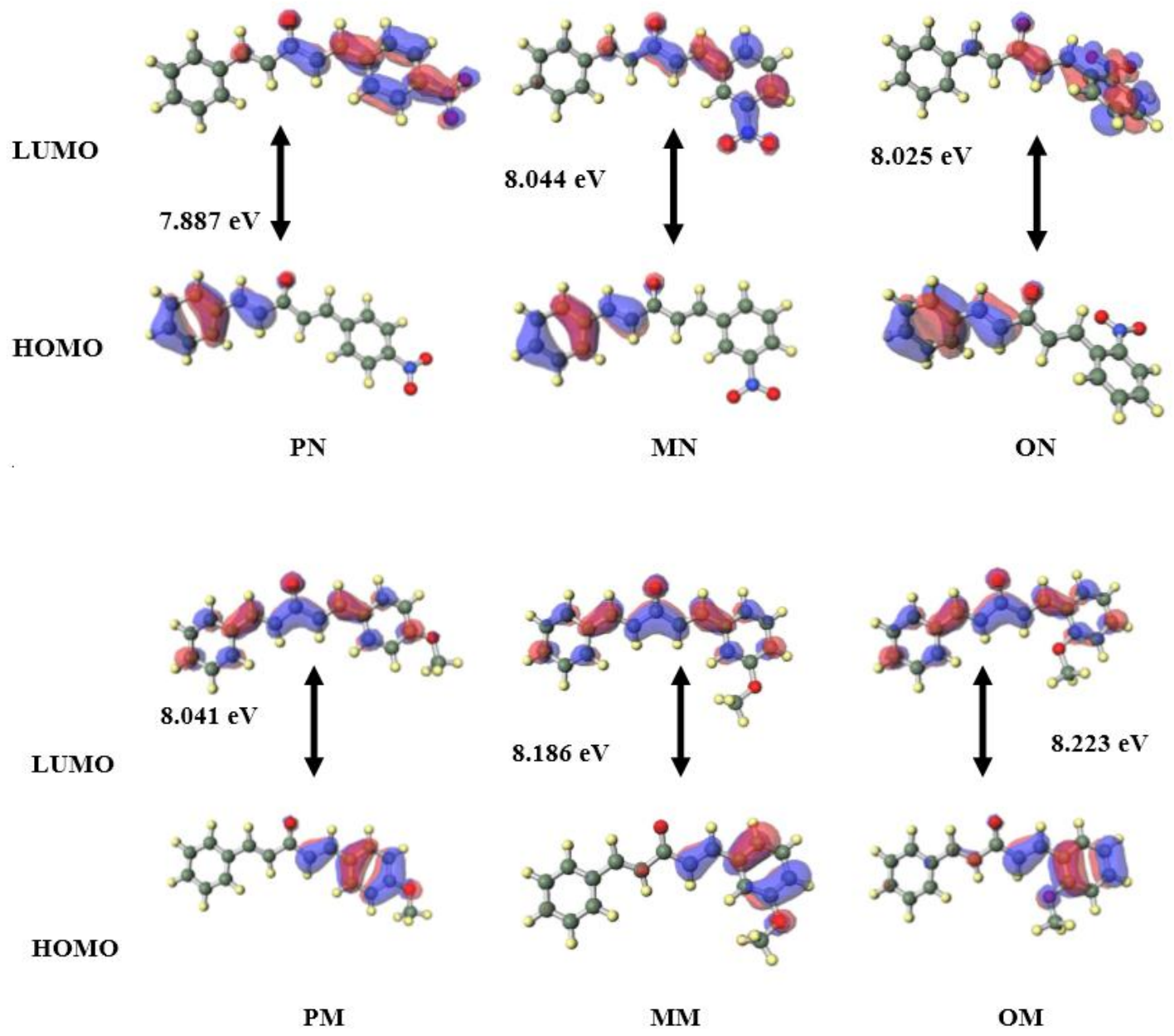

Figure 2. HOMO-LUMO of Donor/Acceptor substituted chalcones

\section{CONCLUSION}

Semi empirical quantum chemical calculations on six Donor/Acceptor substituted chalcones were carried out systematically. The dihedral angle becomes larger in Ortho position substituted chalcone which affects the molecular planarity. As a result the chalcones substituted at the ortho position showed lesser NLO response. In order to design NLO chalcones one need to consider the substitution of Donor/Acceptor at para position of the chalcone molecule.

\section{ACKNOWLEDGEMENT}

Author thank Dr. Ravindra H J for the discussion on the computational result. Author also thank Principal and Management for the support and research facility. 


\section{REFERENCES}

[1] R.W. Boyd, Nonlinear optics, Academic Press, Rochester, New York, 1992.

[2] J. Zyss, Molecular nonlinear optics: Materials, Physics, Devices, Academic Press, Boston, 1994.

[3] D.N. Nikogosyan, Nonlinear optical crystals: A complete survey, Springer, New York, 2005.

[4] H.J. Ravindra, A. John Kiran, K. Chandrasekharan, H.D. Shashikala, S.M.Dharmaprakash, Appl. Phys. B 88, 105-110 (2007)

[5] H.J. Ravindra, K. Chandrasekharan, W.T.A. Harrision, S. M. Dharmaprakash, Appl. Phys. B 94, 503-511 (2009)

[6] J. J. P. Stewart, MOPAC 2016, Stewart Computational Chemistry. Colorado Springs, Co, USA, http://OpenMOPAC.net2016. 
